\title{
The Sex-Dependent Impact of Chronic Clozapine and Haloperidol Treatment on Characteristics of the Metabolic Syndrome in a Rat Model
}

Authors

Affiliations

\author{
M. von Wilmsdorff', M.-L. Bouvier', U. Henning', A. Schmitt" ${ }^{2,3,4}$, T. Schneider-Axmann², ${ }^{2}$, W. Gaebel
}

Department of Psychiatry and Psychotherapy, Medical Faculty, Heinrich-Heine-University, Düsseldorf, Germany Department of Psychiatry, University of Göttingen, Göttingen, Germany

Laboratory of Neuroscience (LIM27), Institute of Psychiatry, University of Sao Paulo, São Paulo - SP, Brazil

${ }^{4}$ Department of Psychiatry, LMU München, München, Germany

\author{
Key words \\ - clozapine \\ - haloperidol \\ - metabolic syndrome \\ - sex-dependence
}

received $\quad 07.02 .2012$

revised 28.06.2012

accepted 11.07.2012

\section{Bibliography}

DOI http://dx.doi.org/

10.1055/s-0032-1321907

Published online ahead of print:

22 August 2012

Pharmacopsychiatry 2013;

46: 1-9

(c) Georg Thieme Verlag KG

Stuttgart - New York

ISSN 0176-3679

\section{Correspondence}

\section{Prof. Dr. A. Schmitt}

Department of Psychiatry

University of Göttingen

Von-Siebold-Straße 5

37075 Göttingen

Germany

Tel.: + 49/551/3910366

Fax: + 49/551/398 952

aschmit@gwdg.de

\section{Abstract}

\section{$\nabla$}

Introduction: An increased risk for metabolic syndrome has been described for patients with psychotic disorders. Antipsychotic drugs possibly contribute to metabolic changes.

Methods: Haloperidol or clozapine was orally fed to male and female Sprague Dawley rats for 12 weeks, and body weight gain, food and water intake were measured. The serum levels of fasting glucose, HbA1c, triglycerides, cholesterol, HDL and LDL, insulin, leptin, adiponectin and ghrelin were determined. Gonadal and perirenal fat pads were removed and weighed.

\section{Introduction}

\section{$\nabla$}

The metabolic syndrome is a cluster of metabolic dysfunctions containing risk factors such as visceral adipositas, dysregulation of glucose metabolism including insulin resistance, atherogenic dyslipidemia and hypertension. It increases the susceptibility for diabetes mellitus type II and cardiovascular diseases associated with a totally increased morbidity and mortality $[1,2]$.

Diverse findings point to a correlation between several psychiatric disorders and the prevalence of metabolic syndrome [3-5]. The mortality of schizophrenia patients due to adipositas, diabetes mellitus and cardiovascular diseases is 2.5fold higher than in the healthy population [6]. But since most patients have a history of longterm antipsychotic treatment, the differentiation between dysfunctions resulting from the disease and drug-induced metabolic dysfunctions is difficult. Thakore et al. [7] reported that - independently of drug medication - schizophrenia patients possess more intra-abdominal fat than control subjects. Drug-naïve schizophrenic patients have a higher prevalence of impaired fasting glucose and a higher level of insulin resistance [8].
Results: We found increased body weight in the male clozapine group, but decreased ones in the male haloperidol group. Clozapine-treated male and female animals had higher fasting glucose, adiponectin, leptin, ghrelin, cholesterol, HDL and LDL levels, whereas haloperidol caused increased levels of insulin and decreased values of HbA1c, cholesterol, HDL and LDL.

Conclusion: Both antipsychotic drugs cause sex-dependent metabolic changes, which are risk factors for the metabolic syndrome, be it hyperinsulinemia under haloperidol treatment or hyperglycemia, hyperleptinemia and hyperlipidemia under clozapine.

Other studies again found no differences between healthy controls and drug-naïve patients in this regard $[9,10]$, for which reason there seems to be lacking agreement whether metabolic changes are drug-induced or disease-related. Another approach [11] reports glucose-insulin homeostasis and lipid profiles in clozapine-induced schizophrenic obesity to differ from those in nonpsychiatric obesity with similar anthropometric parameters, body weight and BMI, also observed with typical agents [12].

According to diverse studies, atypical antipsychotic drugs increase the risk of developing a metabolic syndrome and thus forward the risk for diabetes mellitus and insulin resistance [13]. Correspondingly, De Hert et al. [14] indicated a prevalence of metabolic syndrome of $36 \%$ within schizophrenia patients with antipsychotic medication. Additionally, Lamberti et al. [15] found a respective prevalence in $53.8 \%$ clozapine-treated patients compared to $20.7 \%$ in the general population. Some antipsychotic drugs induce higher food consumption and/or a reduced activity with serious impact on the energy balance. Unlike haloperidol, in schizophrenia patients clozapine increases body weight gain [16] and can cause 
metabolic abnormalities like hyperinsulinemia, hyperlipidemia, hyperleptinemia [17] and dysfunction of the glucose metabolism [18].

Evidence suggests effects of antipsychotic drugs on weight gain [19], metabolic homeostasis and lipid metabolism to be genderdependent [20]: men present a higher level of waist-hip ratio, triglycerides and insulin resistance index than women. This is reflected by animal models, showing that estrogen deficiency results in increased prevalence of cardiovascular diseases in female rats suggesting estrogens to ameliorate the metabolic syndrome as well as insulin resistance [21]. Transient prenatal androgen exposure produces features of the metabolic syndrome in adult female rats [22].

Studies in humans are frequently contradictory and cannot clearly distinguish between disease and treatment-related effects. Experimental research on rodents might support an understanding of the mechanisms underlying antipsychoticinduced metabolic changes which contribute to the development of the metabolic syndrome. Accordant studies were carried out on different rat strains like Wistar [23], including Han-Wis$\operatorname{tar}$ [24], Sprague Dawley (SD) [25] and Long-Evans [26]. In general, in contrast to female Wistar rats, males did not show body weight gain or increased food intake under antipsychotic treatment [27-29]. SD rats differed by exhibiting diet-induced obesity [30] and increased plasma glucose levels [31,32] plus increased body fat deposition [33,34]. Research frequently has focused on metabolic effects in male or female animals. There nevertheless exist some studies directly comparing the effects of antipsychotic drug impact between male and female rats [35]. The aim of our investigation consisted in clarifying the impact of chronic treatment with the typical neuroleptic haloperidol and the atypical antipsychotic clozapine on male and female SD rats. We accordingly investigated body weight gain, food and water intake, fat distribution, and serum levels of parameters correlated with metabolic activity, which is based on the hypothesis that clozapine treatment differs from haloperidol in changing metabolic parameters in male and female SD rats.

\section{Materials and Methods}

$\nabla$

\section{Animals and housing conditions}

All experiments were carried out in accordance with the laws of the local authorities for animal experiments approved by the Landesamt für Natur, Umwelt- und Verbraucherschutz NRW, Recklinghausen (Reference number 9.93.2.10.34.07.227). On postnatal day 21 (PD 21), 30 male and 30 female pups of SD rats (Taconic, Denmark), were separated from their mothers, and housed in groups of 5 same-gender animals with free access to water and ground food pellets. The latter was composed of $89.0 \%$ dry matter, $19.0 \%$ crude protein, $4.0 \%$ crude fat, $6.0 \%$ crude fibre, $7.5 \%$ crude ash with additionally $15 \%$ fat in the dry matter (Altromin Spezialfutter GmbH, Germany). The animals were maintained on a $12: 12 \mathrm{~h} \mathrm{light} /$ dark cycle (lights off at $20.00 \mathrm{~h}$ ) at a temperature of $21^{\circ} \mathrm{C}$ and $60 \%$ humidity. To limit possible individual weight gain effects, the rats of each treatment group emanated from different litters with identical litter size, so each group was equally composed of rats with different parentage.

\section{Treatment with antipsychotics}

The effects of clozapine and haloperidol in adolescent and adult rats differ by substantial pruning and re-organization of the dopamine system occurring from adolescence to adulthood [36]. To avoid these puberty effects, the test started at week 13 (PD 85 ). The healthy animals were individually housed and weighed twice per week (weighing at Monday and Thursday, averaged for a week). Water consumption was calculated for each week and food intake measured each day, averaged for a week, including the loss of ground pellets in the litter. Food conversion ratio FCR (food intake/weight gain), food conversion efficiency FCE [\%] (weight gain/food intake $\times 100$ ) and growth rate [\%/day] (end weight-starting weight) $\times 100 /$ starting weight/test time) were calculated [37]. Based on the study of Kapur et al. [38], who criticized the current chronic dosing of haloperidol in animal studies to be inappropriately low, we used oral doses commensurate to those of the studies of Minet-Ringuet et al. [33,34] who applied dosages correspondend to $50 \%$ of the maximal physiological human dose. Starting at week 13 (PD 85) to week 25 (PD 169), 10 males and 10 females received $1 \mathrm{mg} / \mathrm{kg}$ body weight (BW) haloperidol (Haloneurol ${ }^{\circledR}$, Hexal, Germany) per day in a measured quantity of ground pellets, corresponding to an effective average dose rate of $0.8 \pm 0.03 \mathrm{mg} / \mathrm{kg}$ BW for males and $0.6 \pm 0.08 \mathrm{mg} / \mathrm{kg}$ BW for females. 10 males and 10 females daily received $20 \mathrm{mg} / \mathrm{kg}$ BW clozapine (Leponex ${ }^{\circledR}$, Novartis, Germany), corresponding to $18.5 \pm 0.26 \mathrm{mg} / \mathrm{kg}$ BW for males and $17.7 \pm$ $0.38 \mathrm{mg} / \mathrm{kg}$ BW for females. In a previous study we were able to depict that weekly relative food and water consumption (in relation to $1 \mathrm{~kg}$ body weight) are changing parameters under antipsychotic treatment [29]. The control groups with 10 males and females were merely fed with pure ground pellets. Via dosage and oral application we considered the reported 4-6 times shorter half-life of antipsychotic drugs in rodents compared to humans and intended to avoid stress reactions caused by daily drug injections. Perez-Costas et al. [39] asserted that in rodents appropriate doses of haloperidol in drinking water equalled receptor occupancies comparable to clinical levels, which again does not seem to apply to clozapine. To address these shortcomings, an oral application in ground pellets seemed suitable for investigating chronic treatment periods. Administering olanzapine by minipumps seems to be limited to 2 weeks and is not viable for long-term drug administration [40].

\section{Locomotor activity}

Locomotor activity under antipsychotic drug medication was measured by voluntary wheel running in a rat activity wheel (Tecniplast, Germany) compared to untreated controls. The animals were individually housed in a home cage equipped with a running wheel and free access to food and water for $24 \mathrm{~h}$. A magnetic switch counted complete rotations of the activity wheel.

\section{Determination of metabolic parameters}

Food was removed $12 \mathrm{~h}$ prior anesthesia for determining fasting glucose. On PD 169 (week 25) the animals were anesthetized by pentobarbital (Narcoren, Merial, Germany), blood was collected by aorta puncture, perirenal and gonadal (epididymal and periovarian) fat tissue was removed and immediately weighed. Serum levels of clozapine, $\mathrm{N}$-desmethylclozapine and haloperidol were quantified by HPLC in the biochemical laboratory of the LVR Klinikum Düsseldorf. Briefly, HPLC analyses of clozapine, $N$-desmethylclozapine and haloperidol were performed using equipment from Dionex with the 580 pump and the GINA50 autosampler. For detection, the LC spectrometer Lambda Max 481 (Waters) was used. Drugs were extracted from the blood 
samples with ethyl acetate by rigorous vortexing for $30 \mathrm{~min}$ at room temperature. Prior to extraction, step LY170222 (Lilly) and chlorinated haloperidol analogue (Sigma-Aldrich) were added as internal standards for the quantification of clozapine as well as $N$-desmethylclozapine and for haloperidol, respectively. After ethyl acetate extraction, samples were centrifuged at $2500 \times \mathrm{g}$ for $15 \mathrm{~min}$, the supernatants were collected and evaporated to dryness with a speed vac (SC110A). Residues were dissolved in mobile phase (ammonium acetate ( $\mathrm{pH} 4.5$ ) with $30 \%$ acetonitrile). Separation was performed with a Hypersil CPS (MZ, Germany) combined with Phenomenex security guard cartridges at $36^{\circ} \mathrm{C}$. The flow rate was adjusted to $1 \mathrm{~mL} / \mathrm{min}$ and the adsorbance was measured at $254 \mathrm{~nm}$. Drug quantification was performed with the Chromeleon software (Dionex).

The serum levels of leptin, adiponectin, ghrelin and insulin were determined by a rat leptin and adiponectin ELISA kit (Mediagnost, Germany), by a rat ghrelin Elisa kit (USBiological, USA) and by a rat high range insulin ELISA kit (DRG, Germany) in $\mathrm{ng} / \mathrm{ml}$ or $\mu \mathrm{g} / \mathrm{ml}$ following manufacturer's recommendations. Blood parameters as HbA1c, fasting glucose, total cholesterol, HDL and LDL cholesterol and triglycerides were determined in the clinical laboratory of the LVR Klinikum Düsseldorf.

\section{Statistical analysis}

Statistical analyses were performed with SPSS version 17.0 for Windows. All data are presented as mean \pm SEM and all tests were 2-tailed. Distributions for all dependent variables were examined in both groups using the Kolmogorov-Smirnov test on normality. Our results suggest a normal distribution of the data concluding analysis by parametric tests.

Since 21 dependent variables were examined, the level of significance for the main analyses was adjusted to $\alpha=0.05 /$ $21=0.0024$.
Repeated measures ANOVA with TREATMENT and SEX as between-subject factors and TIME as the repeated measures factor was carried out to elucidate the effects of body weight gain, food and water intake. The results of the activity counts, blood analyses and the Elisa-tests were examined by 2-way ANOVA with the between-subject factors TREATMENT and SEX. For all dependent variables, in case of significant group effects, post hoc tests between the 3 groups with Bonferroni adjusted level of significance for the number of subgroup comparisons ( $\alpha=0.05$ / $6=0.0083$ ) were performed separately for male and female rats. In the case of significant sex-related effects, post-hoc tests between male and female rats were computed for all 3 groups (level of significance Bonferroni adjusted: $\alpha=0.05 / 3=0.0167$ ). (For body weight, food and water intake subgroup analyses were calculated for the mean data over all measurement points.)

A bivariate correlation procedure with Spearman's rho coefficient was carried out to test the relationship between fat tissue (perirenal and gonadal) and weight, as well as fat tissue and the serum levels of leptin, adiponectin, ghrelin and insulin, glucose and insulin with $p \leq 0.05$ as significant.

\section{Results}

$\nabla$

\section{Serum levels of clozapine, $\boldsymbol{N}$-desmethylclozapine and haloperidol}

We found $29.4 \pm 8.4 \mathrm{ng} / \mathrm{mL}$ haloperidol in the serum of male and $31.0 \pm 5.6 \mathrm{ng} / \mathrm{mL}$ in female rats after 12 weeks of oral medication with haloperidol ( $\odot$ Fig. 1); $80.4 \pm 5.6 \mathrm{ng} / \mathrm{mL}$ clozapine and $69.8 \pm 5.5 \mathrm{ng} / \mathrm{mL} N$-desmethylclozapine were detected in male rats and $31.2 \pm 2.4 \mathrm{ng} / \mathrm{mL}$ clozapine and $83.8 \pm 7.3 \mathrm{ng} / \mathrm{mL}$ of the metabolite in females under clozapine treatment. Compared to males, the level of clozapine in females was significantly decreased $(p<0.000001)$, whereas the metabolite was increased. a

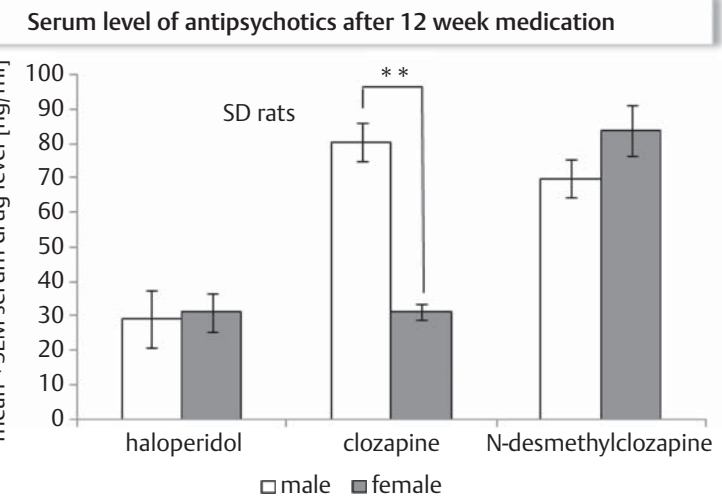

b

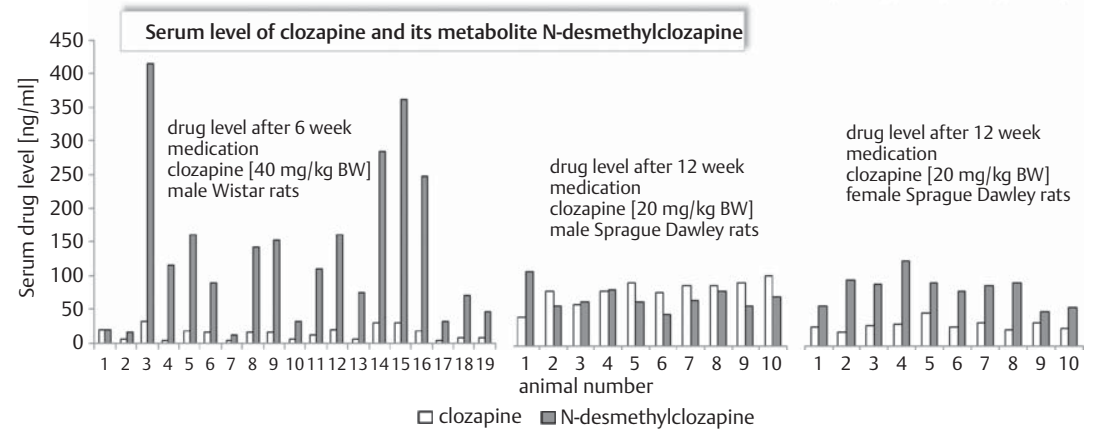

Fig. 1 a Serum level of clozapine, N-desmethylclozapine and haloperidol after oral drug medication ( $n=10$ for all groups). No differences were found between male and female rats treated with haloperidol. Females showed a significant decrease in clozapine level compared to males $\left({ }^{* *} p<0.000001\right)$. Males exhibited a lower level of $\mathrm{N}$-desmethylclozapine, a metabolite of clozapine. b Serum level of clozapine and its metabolite $\mathrm{N}$ desmethylclozapine in single male Wistar and male and female Sprague-Dawley rats by HPLC. Male Wistar and female Sprague-Dawley had a higher serum level of the metabolite, whereas male Sprague-Dawley rats show a higher or equal level of clozapine except animals 1 and 3. 


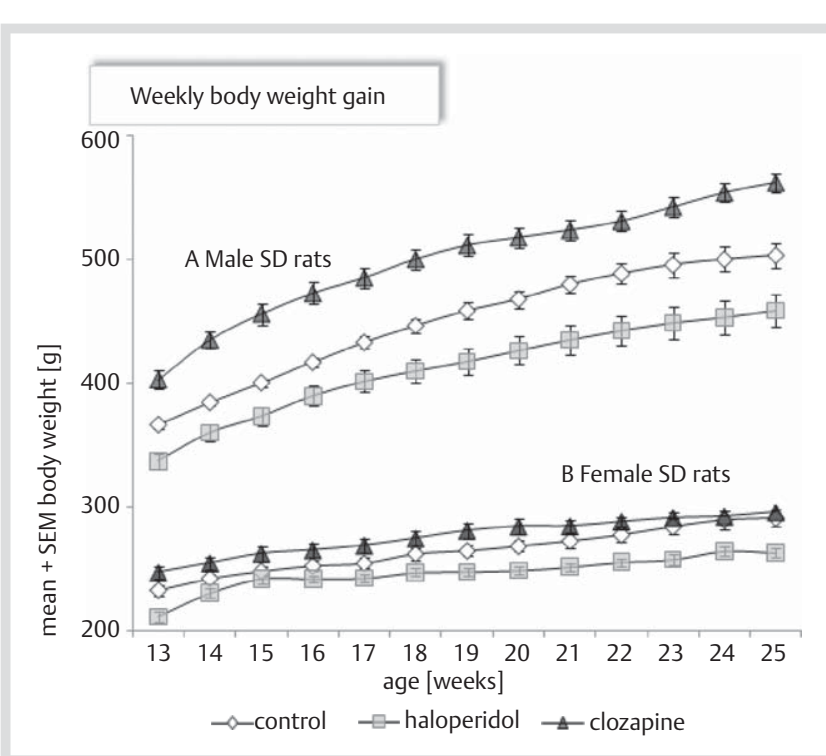

Fig. 2 Weight gain of male and female Sprague-Dawley rats: controls, haloperidol- and clozapine-treated animals ( $n=10$ for all groups). All animals were weighed twice per week. Depicted is the weight after application of the antipsychotic drugs at week 13 to week 25 . The male and female haloperidol group gained less weight than the respective control groups (not significant), whereas the male clozapine group gained significantly more weight than the controls, $p \leq 0.0083$ as significant.

Table 1 Food conversion ratio (FCR), food conversion efficiency (FCE) and growth rate over 12 week medication with clozapine or haloperidol of male and female Sprague-Dawley rats ( $\mathrm{n}=10$ per group).

\begin{tabular}{|llll}
\hline & FCR & FCE & Growth rate \\
male control & $12.8 \pm 0.78$ & $8.10 \pm 0.49$ & $0.39 \pm 0.025$ \\
female control & $22.4 \pm 2.03$ & $4.80 \pm 0.45$ & $0.25 \pm 0.025$ \\
\hline male haloperidol & $12.9 \pm 0.48$ & $7.8 \pm 0.31$ & $0.34 \pm 0.019$ \\
female haloperidol & $24.5 \pm 1.97$ & $4.30 \pm 0.32$ & $0.20 \pm 0.017$ \\
\hline male clozapine & $12.30 \pm 0.29$ & $8.18 \pm 0.20$ & $0.27 \pm 0.011$ \\
\hline female clozapine & $26.9 \pm 1.82$ & $3.89 \pm 0.28$ & $0.20 \pm 0.017$ \\
\hline
\end{tabular}

Single male Wistar [29] and female SD rats had a higher serum level of the metabolite $N$-desmethylclozapine, whereas single male SD rats had a higher or equal level of clozapine, except animals 1 and 3 (see $\bullet$ Fig. 1b).

\section{Weight, food and water intake}

We found a continuous weight gain ( $\bullet$ Fig. 2 ) in the control and drug-treated groups during 12 weeks of drug application TIME $[F(12,648)=804.2, p<0.0024]$. Weight of the animals differed significantly between the groups TREATMENT $[F(2,54)=41.1$, $p<0.0024]$ and there were sex-dependent effects SEX $[F(1$, $54)=1289.7, p<0.0024]$. Male and female rats orally treated with haloperidol had a lower mean body weight than controls and clozapine-treated animals, whereas only male rats under clozapine medication showed significantly higher body weight than controls and haloperidol treated animals (all $p<0.0083$ ). For more details see $\bullet$ Fig. 2.

We found no differences for food conversion ratio $[F(2,54)=1.00$, $p=0.38]$, for food conversion efficiency $[F(2,54)=0.86, p=0.42]$ or growth rate $[F(2,54)=3.1, p=0.055]$, but females differed significantly from males (all $p<0.0167$ ) ( $\diamond$ Table 1 ).

Weekly food $[F(10,540)=11.85, p<0.0024]$ differed over time. Food intake was significantly different for TREATMENT $[F(2,54)=$
$51.2, p<0.0024]$ and SEX $[F(1,54)=410,4, p<0.0024]$, whereas water intake differed significantly for TREATMENT $[F(2,53)=$ $28.4, p<0.0024]$. Food intake was lower both in male and female haloperidol medicated animals compared to controls and clozapine medicated rats, whereas in male and female medicated animals water intake was decreased compared to controls (all $p<0.0083)$. Food intake in females was decreased compared to males (all $p<0.0167$ ), whereas water intake in females under haloperidol was not significantly reduced.

The relative weekly food $[F(10,540)=61.6, p<0.0024]$ and water $[F(10,530)=29.2, p<0.0024]$ intake related to $1 \mathrm{~kg}$ body weight decreased significantly over time and was significantly different for TREATMENT [food: $F(2,54)=23.8, p<0.0024$, water: $[F(2,53)=$ 26.2, $p<0.0024$ ] and SEX [food: $F(1,54)=186.2, p<0.0024$, water: $[F(1,53)=80.7, p<0.0024]$. In males, relative mean food and water intake was significantly lower in the haloperidol than in the control group, whereas in female rats it was decreased in haloperidol-treated compared to clozapine-treated rats and controls (all $p<0.0083$ ). In all 3 groups, relative mean food and water intake was significantly lower in male than in female rats (all $p<0.0167$ ). (For more details see $\odot$ Fig. 3a, b, 4a, b).

\section{Locomotor activity by voluntary wheel running}

We found differences for TREATMENT $[F(2,54)=7.4, p<0.0024]$ and SEX $[F(1,54)=48.7, p<0.0024]$ for locomotor activity tested over 24 h. Control animals showed the highest activity (2697 \pm 296 counts for males and $8155 \pm 1257$ for females) followed by clozapine ( $2516 \pm 290$ for males and $6142 \pm 594$ for females $)$ and haloperidol-treated animals $(1560 \pm 278$ for males and $4091 \pm 767$ for females) confirming the higher sedative effect of haloperidol. However, only the female haloperidol group differed significantly from the controls $(p<0.0083)$. As expected [41], females generally had a higher activity level than males (all $p<0.0167$ ).

\section{Fat tissue and related hormones ( $\bullet$ Table 2)}

Male clozapine-fed rats showed a trend to higher percental fat tissue than controls ( $p>0.0083$ ), and males had a non-significant higher percental fat level than females ( $p>0.0167)$. Significantly positive correlations between endpoint weight and fat tissue were found for controls (males $\rho=0.673, p=0.033$; females $\rho=0.634, p=0.049$ ) and male haloperidol medicated rats $(\rho=0.908, p=0.0003)$.

Male rats treated with clozapine showed a non-significant elevated plasma leptin level compared to controls. The leptin level was significantly decreased in females compared to males in both the clozapine and the haloperidol groups ( $p=0.001$ for both groups) and not significant in the control group ( $p=0.017)$. Male clozapine-fed rats also showed a trend to increased adiponectin $(p=0.042)$ and ghrelin $(p=0.077)$ levels.

The percentage of fat tissue was positively correlated with leptin serum level in the control and haloperidol group (male control $\rho=0.83, p=0.003$; male haloperidol $\rho=0.75, \mathrm{p}=0.013$; female control $\rho=0.83, p=0.003$; female haloperidol $\rho=0.82, p=0.007$ ), but not in the clozapine groups (male clozapine $\rho=0.50, p=0.14$; female haloperidol $\rho=0.10, p=0.78$ ). Fat tissue was negatively correlated with adiponectin serum level in the male haloperidol group $(\rho=-0.66, p=0.038)$.

\section{Glucose and related parameters ( $\odot$ Table 2)}

We found a non-significant elevation of fasting glucose in female rodents treated with clozapine $(p=0.023)$. HbA1c values were 


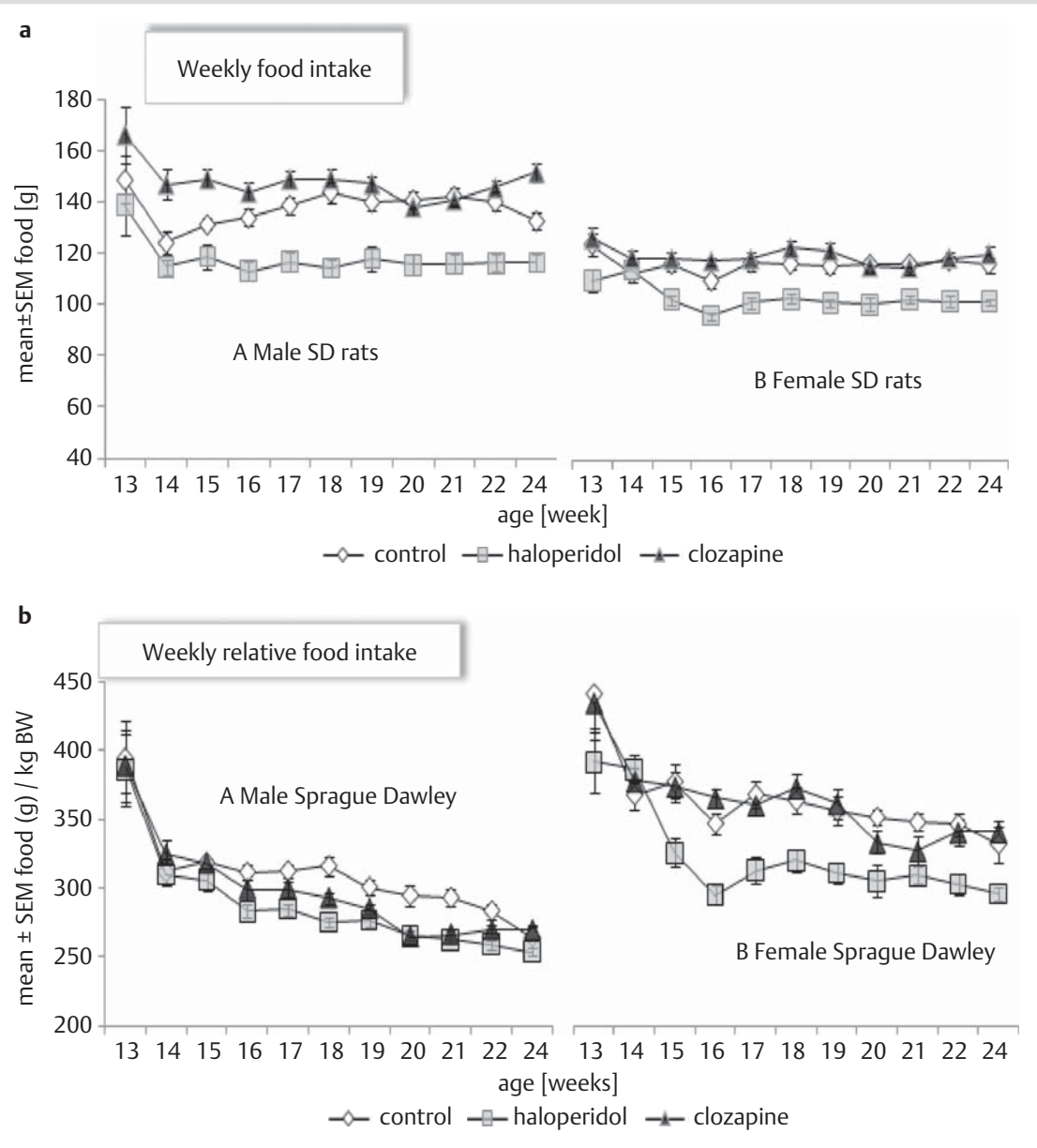

Fig. 3 Weekly food intake of male and female Sprague-Dawley rats: controls, haloperidol and clozapine treated animals ( $n=10$ for all groups). a Weekly food intake: we found no differences between controls and clozapine-medicated rats, although the male clozapine group ate more in general. Animals under haloperidol medication ate significantly less than the control and clozapine group, $p \leq 0.0083$ as significant. b Weekly relative food intake based on $1 \mathrm{~kg} \mathrm{BW}$. All animals showed a decreased food intake from week 13 to week 25 . Animals under haloperidol medication ate significantly less than the control and clozapine group, $p \leq 0.0083$ as significant.

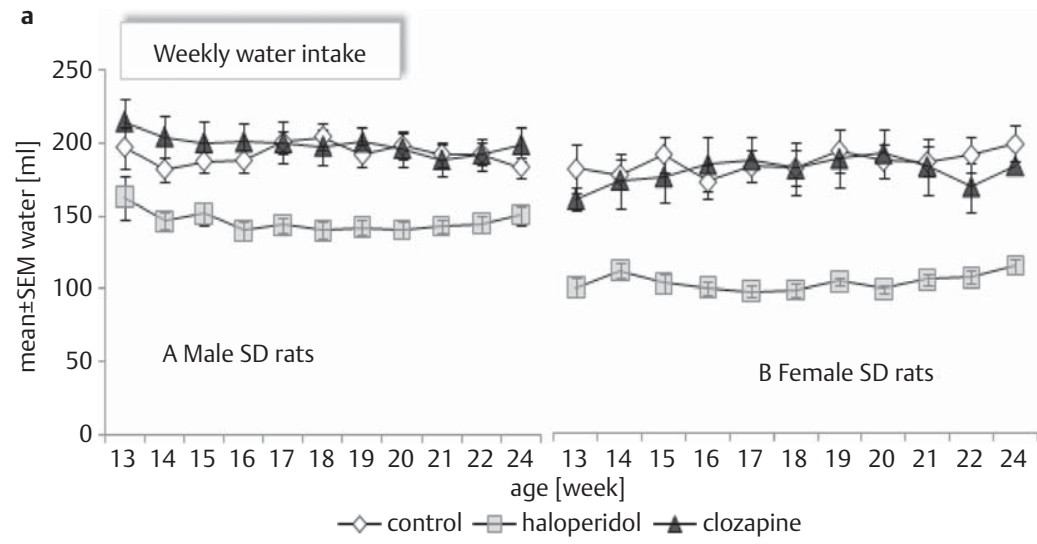

Fig. 4 Weekly water intake of male and female Sprague-Dawley rats: controls, haloperidol and clozapine treated animals ( $n=10$ for all groups). a Weekly water intake: We found no significant differences between control and clozapine-medicated animals. Animals under haloperidol medication drank less than the control and clozapine group, with $p \leq 0.0083$ as significant. $\mathbf{b}$ Weekly relative water intake based on $1 \mathrm{~kg} \mathrm{BW}$ : Male animals showed a lightly decreased water intake from week 13 to week 25 . Animals under haloperidol medication drank less than the control and clozapine group, with $p \leq 0.0083$ as significant.

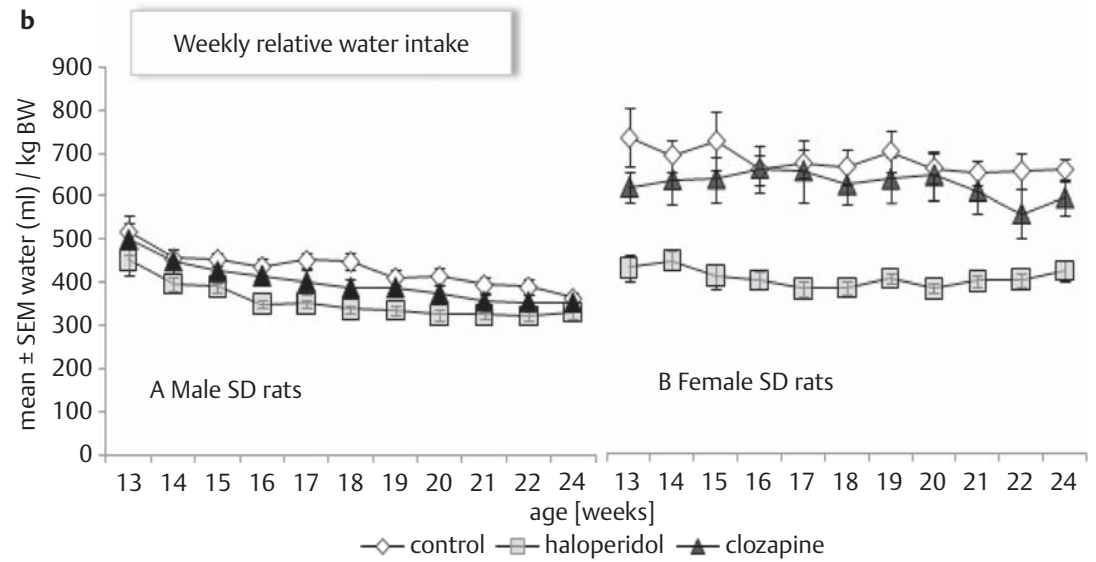


Table 2 Weight of perirenal and gonadal fat pads, level of serum adiponectin, leptin, ghrelin, fasting glucose, insulin, HbA1c and HOMA IR after 12 week of haloperidol and clozapine medication of male and female Sprague Dawley rats ( $n=10$ per group).

\begin{tabular}{|c|c|c|c|c|c|c|c|c|}
\hline & $\begin{array}{l}\text { Fat tissue } \\
\text { [\%BW] }\end{array}$ & $\begin{array}{l}\text { Adiponectin } \\
{[\mu \mathrm{g} / \mathrm{mL}]}\end{array}$ & $\begin{array}{l}\text { Leptin } \\
{[\mathrm{ng} / \mathrm{mL}]}\end{array}$ & $\begin{array}{l}\text { Ghrelin } \\
\text { [ng/mL] }\end{array}$ & $\begin{array}{l}\text { Fasting glucose } \\
{[\mathrm{mg} / \mathrm{dL}]}\end{array}$ & $\begin{array}{l}\text { Insulin } \\
{[\mu g / L]}\end{array}$ & HOMA IR & HbA1c [\%] \\
\hline male control & $5.43 \pm 0.39$ & $5.4 \pm 0.4$ & $2.7 \pm 0.7$ & $4.9 \pm 1.1$ & $200.2 \pm 11.4$ & $7.1 \pm 0.1$ & $0.87 \pm 0.04$ & $3.41 \pm 0.06$ \\
\hline female control & $4.59 \pm 0.39$ & $6.0 \pm 0.4$ & $0.9 \pm 0.1$ & $6.5 \pm 0.6$ & $180.0 \pm 7.8$ & $7.5 \pm 0.3$ & $0.84 \pm 0.05$ & $3.01 \pm 0.05$ \\
\hline male haloperidol & $48.9 \pm 4.54$ & $6.1 \pm 0.4$ & $2.5 \pm 0.4$ & $5.2 \pm 0.8$ & $213.1 \pm 8.1$ & $8.0 \pm 0.5$ & $1.05 \pm 0.07$ & $3.27 \pm 0.08$ \\
\hline female haloperidol & $4.72 \pm 0.38$ & $6.5 \pm 0.4$ & $1.0 \pm 0.1$ & $6.7 \pm 0.9$ & $179.9 \pm 8.2$ & $8.7 \pm 1.2$ & $0.95 \pm 0.1$ & $2.80 \pm 0.02^{*}$ \\
\hline male clozapine & $6.52 \pm 0.30$ & $7.0 \pm 0.5$ & $3.7 \pm 0.5$ & $8.4 \pm 1.2$ & $216.5 \pm 12.0$ & $7.5 \pm 0.3$ & $1.00 \pm 0.07$ & $3.47 \pm 0.09$ \\
\hline female clozapine & $4.86 \pm 0.23$ & $6.9 \pm 0.7$ & $1.2 \pm 0.3$ & $7.8 \pm 0.6$ & $245.6 \pm 29.4$ & $7.3 \pm 0.6$ & $1.11 \pm 0.1$ & $3.01 \pm 0.09$ \\
\hline
\end{tabular}

*Significantly different to the control group $(p<0.0083)$

\begin{tabular}{|lllll|} 
& $\begin{array}{l}\text { Total cholesterol } \\
{[\mathbf{m g} / \mathbf{d L}]}\end{array}$ & $\begin{array}{l}\text { HDLcholesterol } \\
{[\mathbf{m g} / \mathbf{d L}]}\end{array}$ & $\begin{array}{l}\text { LDL-cholesterol } \\
{[\mathbf{m g} / \mathbf{d L}]}\end{array}$ & $\begin{array}{l}\text { Triglycerides } \\
{[\mathbf{m g} / \mathbf{d L}}\end{array}$ \\
\hline male control & $114.0 \pm 3.4$ & $33.2 \pm 0.8$ & $60.2 \pm 2.7$ & $93.0 \pm 7.4$ \\
\hline female control & $118.7 \pm 2.3$ & $37.7 \pm 1.6$ & $70.3 \pm 1.7$ & $53.8 \pm 5.8$ \\
\hline male haloperidol & $104.3 \pm 3.9^{*}$ & $31.8 \pm 0.7$ & $55.7 \pm 4.4$ & $84.4 \pm 8.9$ \\
\hline female haloperidol & $101.7 \pm 5.3$ & $32.3 \pm 1.1$ & $57.3 \pm 4.7$ & $53.2 \pm 6.5$ \\
\hline male clozapine & $127.8 \pm 5.1$ & $33.3 \pm 1.4$ & $75.5 \pm 3.5^{*}$ & $94.7 \pm 6.3$ \\
\hline female clozapine & $150.2 \pm 6.5^{*}$ & $43.5 \pm 1.2^{*}$ & $94.2 \pm 5.4^{*}$ & $62.7 \pm 5.6$ \\
\hline
\end{tabular}

Table 3 Serum levels of total
cholesterol, $\mathrm{HDL}$ - and LDL-choles-
terol, triglycerides after 12 week of
haloperidol and clozapine medica-
tion of male and female Sprague-
Dawley rats ( $\mathrm{n}=10$ per group). significantly decreased in haloperidol-fed rats $(p=0.005)$, and females always had significantly lower levels in all 3 groups (all $p<0.0167)$. Haloperidol-medicated rats showed a trend to increased insulin levels $(p>0.0083)$. The HOMA-IR was elevated either in clozapine or haloperidol groups (not significant, all $p>0.0083)$.

Level of total cholesterol, HDL- and LDL-cholesterol and triglycerides (O Table 3)

There was a TREATMENT effect for total cholesterol $[F(2,54)=$ 31.0, $p<0.0024]$. It was decreased in male haloperidol treated rats compared to controls and increased in female clozapine treated rats compared to the other 2 groups (all $p<0.0083$ ). For HDL-cholesterol there were significant TREATMENT effects $[F(2,52)=14.1, p<0.0024$, increased in the female clozapine group], and also sex-related effects $[F(1,52)=26.9, p<0.0024]$, since in clozapine-medicated rats females showed higher values than males $(p<0.0167)$. For LDL cholesterol, there were significant TREATMENT effects, as well $[F(2,52)=27.3, p<0.0024]$. In male and female rats, values in the clozapine group were increased in comparison to the haloperidol and the control groups (all $p<0.0083$ ).

There was a significant sex effect for triglyceride levels $[F(1,54)=$ $37.2, p<0.0024]$. Males always had a significant higher amount of triglycerides than females ( $p=0.001$ for controls, $p=0.011$ for haloperidol and $p=0.001$ for clozapine medication).

\section{Discussion}

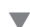

After 6 weeks, the results of SD compared to Wistar rats [26] yielded a lower food conversion ratio (FCR), higher food conversion efficiency (FCE) and higher growth rate (GR) in SD rats (unpublished data). This concurs with the findings of Kühn et al. [42], namely that higher growth rate in SD rats is completely attributed to a significant higher food conversion compared to Wistar rats, since food intake of both groups was comparable. SD rats moreover showed higher body weight, fasting glucose, fast- ing insulin and HOMA-IR than Wistar rats [43]. The study of Chovan et al. [44], demonstrated different pharmacokinetics and metabolic profiles. Gao et al. [45] found intrinsic metabolic differences like higher individualized metabolic variations in fasting and feeding states, and a stronger ability to recover from an altered metabolic profile with less hepatic injury in SD compared to Wistar rats. A possible explication of the metabolic differences between the different strains and sexes could be the fact that clozapine had a lower metabolic conversion rate in male SD rats: male Wistar [29] and female SD rats, both exhibiting no robust weight gain or fat deposition, showed a high level of the metabolite N-desmethyl-clozapine, whereas male SD rats had an equal or higher level of clozapine. In patients, $\mathrm{N}$-desmethylclozapine has higher half-life elimination and possibly contributes significantly to the atypical effects of clozapine treatment by blocking the same receptors. Hence in summary we regard SD as a more appropriate rat model for metabolic changes than Wistar rats.

In our study we found significant alterations of body weight in the drug-treated group of male SD rats. Food and water intake was decreased in the haloperidol group. The reducing effect of orally applied haloperidol on body weight of male and female rats can at least partially be explained by lower food and water intake and the sedative quality of the drug. But food and water intake cannot solely be the limiting factor of body weight gain. Female haloperidol-treated animals absorbed significantly less food without a relevant impact on body weight. Male rats treated with clozapine had a slightly lower food and water intake compared to controls with increased body weight and fat weight, but no increased FCR, FCE or growth rate (see $\odot$ Table 1 ). We therefore assume the increased body weight of male SD rats under clozapine medication to probably result from an increased fat deposition, although our findings show only a trend toward increased fat tissue. Moreover, end weight and fat tissue are positively correlated only in controls and rats exposed to haloperidol suggesting that a high amount of fat in clozapine treated rats is not removed. The study of Jassim et al. [46] could show that clozapine is primarily associated with effects on carbohydrate 
and lipid metabolism in metabolically active peripheral tissues prior to the development of drug-induced weight gain. According to other studies [47] a direct and potent effect of clozapine on adipose tissue is not an important mechanism to induce metabolic disturbances in humans. In animal models antipsychoticinduced metabolic side effects are not necessarily associated with pronounced weight gain [33]. These findings comply with a clinical study showing that antipsychotic treatment can cause impaired glucose regulation independently of adiposity [48]. Several studies found no clozapine effect on food intake and body weight in male and female rats. For example, Choi et al. [25] applied the drug by constant infusion $(10 \mathrm{mg} / \mathrm{kg} /$ day $)$ on 60 -day-old SD rats for 11 days. Cooper et al. [24] found no weight gain and hyperphagia but enhanced adiposity in female Wistar rats after clozapine injections (i.p., b.i.d.) over 21 days. In our study we used a 3-month treatment period possibly useful to find alterations in weight gain. Additionally, instead of daily injections, we offered food prepared with antipsychotics in order to establish a regular supply comparable to the human one with several daily doses to ensure a durable or constant blood level of antipsychotics [34]. Such as human patients treated with antipsychotics, not all rats exhibited abnormal weight gain and/ or metabolic changes. But our sample might be too small to find significantly increased fat content after treatment. Despite their controlled origin, our animals showed a widespread variability in response to drug medication. Considering the genetic background, it has been revealed that different body weight phenotypes within outbred SD populations react differently to the development of drug- or diet-induced obesity via altered anabolic features in liver and hypothalamus [49] and can be obesityprone (OP) or obesity-resistant (OR) [50]. The underlying mechanisms are yet not known and need further investigation. Furthermore, haloperidol medication led to reduced water intake in rats, which possibly aggravates the observed symptoms of apathy and tiredness. Mechanisms underlying druginfluenced drinking behaviour and their role in metabolism are only dissatisfyingly investigated. There exist hints [51] on a modulating interaction between leptin and nitric oxide, which seems to have a thirst- and appetite-reducing effect itself [52].

Minet-Ringuet et al. [33] showed that in male SD rats haloperidol and olanzapine did not modify caloric intake or body weight, but significantly increased adipose tissue and circulating leptin. We found a marginal effect of haloperidol on adiponectin but not on the leptin level. Clozapine-treated rats showed an increased ghrelin level, whereas the leptin and adiponectin levels were also elevated in males under clozapine medication. As expected, leptin levels were positively correlated with percent fat mass in controls and haloperidol groups, but surprisingly not in the clozapine groups, suggesting that the leptin level in clozapine-medicated rats not only depends on fat mass, but possibly also on ghrelin or adiponectin secretion. Normally, ghrelin rises during fasting phases, but clozapine seems to increase plasma ghrelin independently of the nutritional state [53,54]. Ghrelin again enhances white adipose tissue leptin production by a direct receptor-mediated effect [55]. Obese rats generally have reduced adiponectin serum levels, but clozapine might additionally influence adiponectin regulation regardless of fat content, as verified in human patients [56]. Hyperleptinemia was independently associated with clozapine treatment in human patients [57]. According to these results we found higher leptin and adiponectin levels in male rats under clozapine medication.
Clozapine did not affect fat content in female rats. Respectively medicated, they in fact suffered from hyperglycemia which led to an elevated HOMA-IR. Contrastingly, the increased HOMA-IR in the haloperidol group was not caused as a result of high glucose level but rather by high insulin level. Following changes in glucose metabolism for at least 8 weeks, HbA1c represents the blood level of glucose, which was not used in metabolism. But decreased HbA1c-level under haloperidol medication in female rats, less pronounced in males, is probably due to the anemia (decreased levels of erythrocytes, hemoglobin and hematocrit; unpublished data) in these animals. As to humans, Emsley et al. [58] found an elevated HbA1c level in schizophrenia patients treated with haloperidol. In human patients haloperidol causes only minimal changes in erythrocyte counts [59] thus exacerbating comparisons between human conditions and animal models.

Dysglycemia-like pathological fasting glucose levels or insulin resistance are risk factors for the metabolic syndrome, and impaired glucose tolerance can be found in clozapine- but not halperidol-treated patients [60]. Fortifying our results in an animal model, Tulipano et al. [32] proved clozapine to induce sexrelated alterations of glucose homeostasis with the significant highest serum glucose level and reduced insulin sensitivity in female rats without effects on food intake. Impaired peripheral glucose use and/or increased glucose output from the liver might be responsible for the hyperglycemia in clozapine-treated females. It has indeed been shown that clozapine impairs glucose metabolism not by direct induction of insulin resistance but rather acts via an increase in glucagon secretion [61]. Thus clozapine possibly stimulates hepatic glucose production. A limiting factor of our study is the fact that the experimental setup is not well designed for detecting antipsychotic-induced glucose dysregulation, which would require that the system is challenged, e.g., by glucose tolerance tests and/or by hyperinsulinemic clamp experiments. However, the mechanisms underlying the resistance of male rats to clozapine-induced hyperglycemia need to be further investigated. As outlined by Best et al. [62], clozapine has no effect on basal insulin release but significantly inhibits glucose-induced secretion. In contrast, the depolarizing action on pancreatic $\beta$-cell-membrane of haloperidol might be expected to enhance insulin release. Along with our findings we suppose haloperidol and clozapine to differently influence the glucose metabolism.

Total cholesterol and LDL-cholesterol were increased in both clozapine groups, but decreased in the haloperidol groups. HDLcholesterol was enhanced in females medicated with clozapine and reduced in the female haloperidol group. Dyslipidemia is a risk factor for the metabolic syndrome, and increased total cholesterol plus LDL-cholesterol are involved in the etiology of coronary heart diseases. Our findings of dyslipidemia match clinical studies, showing that clozapine is associated with an increase in cholesterol levels [63] in schizophrenia patients.

We did not find significant changes in the level of triglycerides. Compared to cholesterol, triglyceride concentrations show a high variability and can be nutritionally affected. Kimura et al. [64] discovered that time and intake amounts of fat influence triglyceride levels. So we assume that triglyceride levels, which presumably remain unchanged by antipsychotic drug application, are caused by the animals' overnight fasting, the time period in which rats normally have the main food intake. 
However, our study entails some limitations. The depicted results appear to critically depend on the experimental procedures, especially in terms of drug application, duration of medication, rat strains used, diet and drug doses applied. Drug administration via feeding compared to gavage or injections is the least-invasive application procedure, reduces handling stress and allows the exact determination of drug, food and water intake. But oral application requires social isolation of the test animals. Social isolation, even if regarded as a minor stress factor, can change parameters of the hypothalamo-pituitary-adrenal axis (HPA axis) like corticosterone release, involved in the glucose homeostasis [65], inhibit oxidative metabolism [66], and stimulate hippocampal estradiol synthesis [67]. These effects need further investigation. In order to determine the fat content of each animal, we excised the perirenal (retroperitoneal), the epididymal and periovarian fat pads, but not the mesenteric and subcutaneous fat, which hardly can be completely removed [68]. Although this omission admittedly curtails our analysis, the procedure avoids erroneous fat removal. To determine fasting glucose, our test animals fasted $12 \mathrm{~h}$ (dark period) before blood withdrawal. Fasting [69] can decrease the degree of obesity induced by high fat diet, can change the effect of leptin or insulin, and can cause serious disturbance of neuroendocrine peptides. Our animals were fed with ground pellets containing $15 \%$ additional fat in the dry matter. Weight gain, fat mass, plasma glucose, cholesterol, triglyceride, leptin and insulin increase dose-dependently with increased intake of dietary fat [70]. The impact of these parameters on the results of our study needs further investigation.

Despite these limitations, we could show significant sex- and treatment-dependent differences. But the underlying mechanisms, mainly the effect of estrogens, remain unclear and should be the target of further studies. In our study we have disregarded the estrus of females, although activity, food and water intake, and body weight vary across the ovarian cycle [71].

In summary, our animal model suggests chronic antipsychotic drug medication over a 12-week test period to possibly influence body weight gain, fat tissue metabolism and the level of related hormones, to cause dyslipidemia, hyperglycemia or hyperinsulinemia with sex-related impact in SD rats. All these effects are risk factors for the metabolic syndrome. Considering the explorative design for activity counts, blood analyses and ELISA tests, our findings offer no conclusive evidence for a causal relationship resulting from multiple testing. Thus this study should be replicated in an independent larger sample to confirm our relevant positive findings. And it should be supplemented by collateral investigations in human patients to clarify effects related to the metabolic syndrome.

\section{Acknowledgements}

We thank Dr. A. Treiber, former director of the TVA, HeinrichHeine-Universität, Düsseldorf and her assistants for excellent support regarding the animal experiments. Dr. M. Jänner, LVR Klinikum, Düsseldorf, kindly shared her expertise in statistical analysis of our results. Margit Geisler, LVR Klinikum, Düsseldorf, kindly carried out the ELISA tests and the determination of serum drug levels by HPLC.

\section{Conflict of Interest}

$\nabla$

The authors declare that there is no conflict of interest.

\section{References}

1 Henderson DC. Schizophrenia and comorbid metabolic disorders. J Clin Psychiatry 2005; 66 (Suppl 6): 11-20

2 Sacks FM. Metabolic syndrome: epidemiology and consequences. J Clin Psychiatry 2004; 65 (Suppl 18): 3-12

3 Jones $L E$, Carney $C P$. Increased risk for metabolic syndrome in persons seeking care for mental disorders. Ann Clin Psychiatry 2006; 18: $149-155$

4 McEvoy JP, Meyer JM, Goff DC et al. Prevalence of the metabolic syndrome in patients with schizophrenia: baseline results from the Clinical Antipsychotic Trials of Intervention Effectiveness (CATIE) schizophrenia trial and comparison with national estimates from NHANES III. Schizophr Res 2005; 80: 19-32

5 Casey DE, Haupt DW, Newcomer JW et al. Antipsychotic-induced weight gain and metabolic abnormalities: implications for increased mortality in patients with schizophrenia. J Clin Psychiatry 2004; 65 (Suppl 7): 4-18

6 Guo JJ, Keck PE Jr, Corey-Lisle PK et al. Risk of diabetes mellitus associated with atypical antipsychotic use among patients with bipolar order: A retrospective, population-based, case-control study. J Clin Psychiatry 2006; 67: 1055-1061

7 Thakore JH, Mann JN, Vlahos I et al. Increased visceral fat distribution in drug-naïve and drug-free patients with schizophrenia. Int J Obesity 2002; 26: 137-141

8 Ryan MC, Collins $P$, Thakore JH. Impaired fasting glucose tolerance in first-episode, drug-naïve patients with schizophrenia. Am J Psychiatry 2003; 160: 284-289

9 Arranz B, Rosel P, Ramirez $N$ et al. Insulin resistance and increased leptin concentrations in noncompliant schizophrenia patients but not in antipsychotic-naïve first-episode schizophrenia patients. J Clin Psychiatry 2004; 65: 1335-1342

10 Sengupta S, Parrilla-Escobar MA, Klink $R$ et al. Are metabolic indices different between drug-naïve first-episode psychosis patients and healthy controls? Schizophr Res 2008; 102: 329-336

$11 \mathrm{Wu}$ MK, Huang CY, Liou YJ et al. Glucose-insulin homeostasis, lipid profiles and GH-IGF-IGFBP axis in clozapine-treated schizophrenic obesity versus non-psychiatric obesity. Int J Obesity 2008; 32: 436-442

12 Baptista T, La Cruz A, Mendoza $S$ et al. Endocrine and metabolic abnormalities involved in the obesity associated to typical antipsychotic drug administration. Pharmacopsychiatry 2001; 34: 223-231

13 Bergman RN, Ader M. Atypical antipsychotics and glucose homeostasis. J Clin Psychiatry 2005; 66: 504-514

14 De Hert MA, van Winkel R, van Eyck $D$ et al. Prevalence of the metabolic syndrome in patients with schizophrenia treated with antipsychotic medication. Schizophr Res 2006; 83: 87-93

15 Lamberti JS, Olson D, Crilly JF et al. Prevalence of the metabolic syndrome among patients receiving clozapine. Am J Psychiatry 2006; 163: 1273-1276

16 Brixner DI, Said Q, Corey-Lisle PK et al. Naturalistic impact of secondgeneration antipsychotics on weight gain. Am Pharmacother 2006; 40: 626-632

17 Melkersson KI, Dahl ML. Relationship between levels of insulin or triglycerides and serum concentrations of the atypical antipsychotics of clozapine and olanzapine in patients on treatment with therapeutic doses. Psychopharmacology 2003; 170: 157-166

18 Henderson DC, Cagiero E, Copeland PM et al. Glucose metabolism in patients with schizophrenia treated with atypical antipsychotic agents: a frequently sampled intravenous glucose tolerance test and minimal model analysis. Arch Gen Psychiatry 2005; 62: 19-28

19 Ascher-Svanum H, Stensland $M$, Zhao Z et al. Acute weight gain, gender, and therapeutic response to antipsychotics in the treatment of patients with schizophrenia. BMC Psychiatry 2005; 5: 3-16

$20 \mathrm{Wu} R R$, Zhao JP, Zhai JG et al. Sex difference in effects of typical and atypical antipsychotics on glucose-insulin homeostasis and lipid metabolism in first-episode schizophrenia. J Clin Psychopharmacol 2007; 27: 374-379

21 Shehata M, Kamel MA. Protective effect of antioxidant adjuvant treatment with hormone replacement therapy against cardiovascular diseases in ovariectomized rats. Endocr Regul 2008; 42: 69-75

22 Demissie M, Lazic M, Foecking EM et al. Transient prenatal androgen exposure produces metabolic syndrome in adult female rats. Am J Physiol Endocrinol Metab 2008; 295: E262-E268 
23 Pouzet B, Mow T, Kreilgaard $M$ et al. Chronic treatment with antipsychotics in rats as a model for antipsychotic-induced weight gain in human. Pharmacol Biochem Behav 2003; 75: 133-140

24 Cooper GD, Harrold JA, Halford JCG et al. Chronic clozapine treatment in female rats does not induce weight gain or metabolic abnormalities but enhances adiposity: Implications for animal models of antipsychotic-induced weight gain. Prog Neuropsychopharmacol Biol Psychiatry 2008; 32: 428-436

25 Choi S, Disilvio B, Unangst J et al. Effect of chronic infusion of alanzapine and clozapine on food intake and body weight gain in male and female rats. Life Sci 2007; 81: 1024-1030

26 Wiley JL. Antipsychotic-induced suppression of locomotion in juvenile, adolescent and adult rats. Eur J Pharmacol 2008; 578: 216-221

27 Baptista T, Parada M, Hernandez L. Long term administration of some antipsychotic drugs increases body weight and feeding in rats. Are D2 dopamine receptors involved? Pharmacol Biochem Behav 1987; 27: 399-405

28 Baptista T, de Baptista EA, Ying Kin NMK et al. Comparative effects of the antipsychotics sulpiride or risperidone in rats I. bodyweight, food intake, body composition, hormones and glucose tolerance. Brain Res 2002; 957: 144-151

29 Wilmsdorff $M$, Bouvier $M L$, Henning $U$ et al. The impact of antipsychotic drugs on food intake and body weight and on leptin levels in blood and hypothalamic ob-r leptin receptor expression in wistar rats. Clinics 2010; 65: 1-10

30 Mercer JG, Archer ZA. Diet-induced obesity in the Sprague-Dawley rat: dietary manipulations and their effect on hypothalamic neuropeptide energy balance systems. Biochem Soc Trans 2005; 33: 1068-1072

31 Assié MB, Carilla-Durand E, Bardin L et al. The antipsychotics clozapine and olanzapine increase plasma glucose and corticosterone levels in rats: comparison with aripiprazole, ziprasidone, bifeprunox and F15063. Eur J Pharmacol 2008; 592: 160-166

32 Tulipano G, Rizzetti C, Bianchi I et al. Clozapine-induced alteration of glucose homeostasis in the rat: contribution of hypothalamic-pituitary-adrenal axis activation. Neuroendocrinology 2007; 85: 61-70

33 Minet-Ringuet J, Even PC, Goubern $M$ et al. Long term treatment with olanzapine mixed with the food in male rats induces body fat deposition with no increase in body weight and no thermic alteration. Appetite 2006; 46: 254-262

34 Minet-Ringuet J, Even PC, Lacroix $M$ et al. A model for antipsychoticinduced obesity in the male rat. Psychopharmacology 2006; 187: 447-454

35 Boyda HN, Tse L, Procyshyn RM et al. Preclinical models of antipsychotic drug-induced metabolic side effects. Trends Pharmacol Sci 2010; 31: 484-497

36 Wiley JL, Evans RL. Evaluation of age and sex differences in locomotion and catalepsy during repeated administration of haloperidol and clozapine in adolescent and adult rats. Pharmacol Res 2008; 58: 240-246

37 Lemieux H, Blier P, Dutil JD. Do digestive enzymes set a physiological limit on growth rate and food conversion efficiency in the Atlantic cod (Gadus morhua)? Fish Physiol Biochem 1999; 20: 293-303

38 Kapur S, Wadenberg ML, Remington $G$. Are animal studies of antipsychotics appropriately dosed? Lessons from the bedside to the bench. Can J Psychiatry 2000; 45: 241-246

39 Perez-Costas E, Guidetti P, Melendez-Ferro $M$ et al. Neuroleptics and animal models: Feasibility of oral treatment monitored by plasma levels and receptor occupancy assays. J Neural Transm 2008; 115: 745-753

40 Remington $G$, Mann $S$, McCormick $P$ et al. Modeling chronic olanzapine exposure using osmotic minipumps: pharmacological limitations. Pharmacol Biochem Behav 2011; 100: 86-89

41 Lightfoot JT. Sex hormones regulation of rodent physical activity: a review. Int J Biol Sci 2008; 4: 126-132

42 Kühn ER, Bellon K, Huybrechts L et al. Endocrine differences between the Wistar and Sprague Dawley laboratory rat: influence of cold adaption. Horm Metabol Res 1983; 15: 491-498

43 Cacho J, Sevillano J, de Castro J et al. Validation of simple indexes to assess insulin sensitivity during pregnancy in Wistar and Sprague Dawley rats. Am J Physiol Endocrinol Metab 2008; 295: E1269-E1276

44 Chovan JP, Yu E, Ring SC. Metabolic differences between Sprague Dawley and Wistar Han male rats. ISSX Poster and oral presentations 2007

45 Gao X, Zhao A, Zhou $M$ et al. GC/MS-based urinary metabolomics reveals systematic differences in metabolism and ethanol response between Sprague-Dawley and Wistar rats. Metabolomics 2011; 7: 363-374

46 Jassim G, Skrede S, Vázques MJ et al. Acute effects of orexigenic antipsychotic drugs on lipid and carbohydrate metabolism in rat. Psychopharmacology 2012; 219: 783-794
47 Sertié AL, Suzuki AM, Sertié RAL et al. Effects of antipsychotics with different weight gain liabilities on human in vitro models of adipose tissue differentiation and metabolism. Prog Neuro-Psychopharmacol Biol Psychiatry 2011; 35: 1884-1890

48 Newcomer JW, Haupt DW, Fucetola $R$ et al. Abnormalities in glucose regulation during antipsychotic treatment of schizophrenia. Arch Gen Psychiatry 2002; 59: 337-345

49 Jang I, Hwang D, Lee J et al. Physiological difference between dietary obesity-susceptible and obesity-resistant Sprague Dawley rats in response to moderate high fat diet. Exp Anim 2003; 52: 99-107

50 Leibowitz KL, Chang GQ Pamy PS et al. Weight gain model in prepupertal rats: prediction and phenotyping of obesity-prone animals at normal body weight. Int J Obesity 2007; 31: 1210-1221

51 Yang SJ, Denbow DM. Interaction of leptin and nitric oxide on food intake in broilers and Leghorns. Physiol Behav 2007; 92: 651-657

52 Calapai G, Caputi AP. Nitric oxide and drinking behavior. Regul Pept 1996; 66: 117-121

53 Murashita M, Kusumi I, Hosoda $\mathrm{H}$ et al. Acute administration of clozapine concurrently increases blood glucose and circulating plasma ghrelin levels in rats. Psychoneuroendocrinology 2007; 32: 777-784

54 Esen-Danaci A, Sarandöl A, Taneli $F$ et al. Effects of second generation antipsychotics on leptin and ghrelin. Prog Neuropsychopharmacol Biol Psychiatry 2008; 32: 1434-1438

55 Giovambattista A, Piermaria J, Suescun MO et al. Direct effect of ghrelin on leptin production by cultured rat white adipocytes. Obesity 2006; 14: 19-38

56 Hanssens $L$, van Winkel $R$, Wampers $M$ et al. A cross-sectional evaluation of adiponectin plasma levels in patients with schizophrenia and schizoaffective disorder. Schizophr Res 2008; 106: 308-314

57 Hägg S, Söderberg S, Ahrén B et al. Leptin concentrations are increased in subjects treated with clozapine or conventional antipsychotics. J Clin Psychiatry 2001; 62: 843-848

58 Emsley $R$, Turner JH, Schronen $J$ et al. Effects of quetiapine and haloperidol on body mass index and glycaemic control: a long-term, randomized, controlled trial. Int J Neuropsychopharmacol 2005; 8: $175-182$

59 Flanagan RJ, Dunk L. Haematological toxicity of drugs used in psychiatry. Hum Psychopharmacol Clin Exp 2008; 23: 27-41

60 Chae BJ, Kang BJ. The effect of clozapine on blood glucose metabolism. Hum Psychopharmacol 2001; 16: 265-271

61 Smith GC, Chaussade C, Vickers $M$ et al. Atypical antipsychotic drugs induce derangements in glucose homeostasis by acutely increasing glucagon secretion and hepatic glucose output in the rat. Diabetologica 2008; 51: 2309-2317

62 Best L, Yates AP, Reynolds GP. Actions of antipsychotic drugs on pancreatic $\beta$-cell function: contrasting effects of clozapine and haloperidol. J Psychopharmacol 2005; 19: 597-601

63 Lindenmayer JP, Czobor P, Volavka J et al. Changes in glucose and cholesterol levels in patients with schizophrenia treated with typical or atypical antipsychotics. Am J Psychiatry 2003; 160: 290-296

64 Kimura F, Natsusaka J, Endo $Y$ et al. Effect of dose of fat and fat content of the maintenance diet on postprandial serum triglyceride in rats. J Oleo Science 2004; 53: 515-520

65 Stairs DJ, Prendergast MA, Bardo MT. Environmental-induced differences in corticosterone and glucocorticoid receptor blockade of amphetamine self-administration in rats. Psychopharmacology 2011; 218: 293-301

66 Zhuravliova E, Barbakadze T, Zaalishvili et al. Social isolation in rats inhibits oxidative metabolism, decreases the content of mitochondrial K-Ras and activates mitochondrial hexokinase. Behav Brain Res 2009; 205: 377-383

67 Munetsuna E, Hattori M, Komatsu $S$ et al. Social isolation stimulates hippocampal estradiol synthesis. Biochem Biophys Res Commun 2009; 379: 480-484

68 Héliès JM, Diane A, Langlois A et al. Comparison of fat storage between Fischer 344 and obesity-resistant Lou/C rats fed different diets. Obesity Res 2005; 13: 3-10

$69 \mathrm{Wu} B, \mathrm{Du} Y$, Liu $\mathrm{C}$ et al. Effect of repeated fasting/reffeding on body weight weight control and energy balance regulation in rats. Wei Sheng Yan Jiu 2010; 39: 601-605

70 Ghibaudi L, CookJ, Farley $C$ et al. Fat intake affects adiposity, comorbidity factors, and energy metabolism of Sprague Dawley rats. Obesity Res 2002; 10: 956-963

71 Eckel LA, Houpt TA, Geary N. Spontaneous meal patterns in female rats with and without access to running wheels. Physiol Behav 2000; 70: $397-405$ 